\title{
Biosynthesis of tovarol and other sesquiterpenoids in Thapsia laciniata Rouy
}

Andersen, Trine Bundgaard; Rasmussen, Silas Anselm; Christensen, Søren Brøgger; Simonsen, Henrik Toft

Published in:

Phytochemistry

Link to article, DOI:

10.1016/j.phytochem.2018.10.027

Publication date:

2019

Document Version

Peer reviewed version

Link back to DTU Orbit

Citation $(A P A)$ :

Andersen, T. B., Rasmussen, S. A., Christensen, S. B., \& Simonsen, H. T. (2019). Biosynthesis of tovarol and other sesquiterpenoids in Thapsia laciniata Rouy. Phytochemistry, 157, 168-174.

https://doi.org/10.1016/j.phytochem.2018.10.027

\section{General rights}

Copyright and moral rights for the publications made accessible in the public portal are retained by the authors and/or other copyright owners and it is a condition of accessing publications that users recognise and abide by the legal requirements associated with these rights.

- Users may download and print one copy of any publication from the public portal for the purpose of private study or research.

- You may not further distribute the material or use it for any profit-making activity or commercial gain

- You may freely distribute the URL identifying the publication in the public portal

If you believe that this document breaches copyright please contact us providing details, and we will remove access to the work immediately and investigate your claim. 


\section{Graphical abstract}

2 The biosynthesis of sesquiterpenoids in Thapsia laciniata Rouy was investigated, and three terpene

3 synthases was characterized.

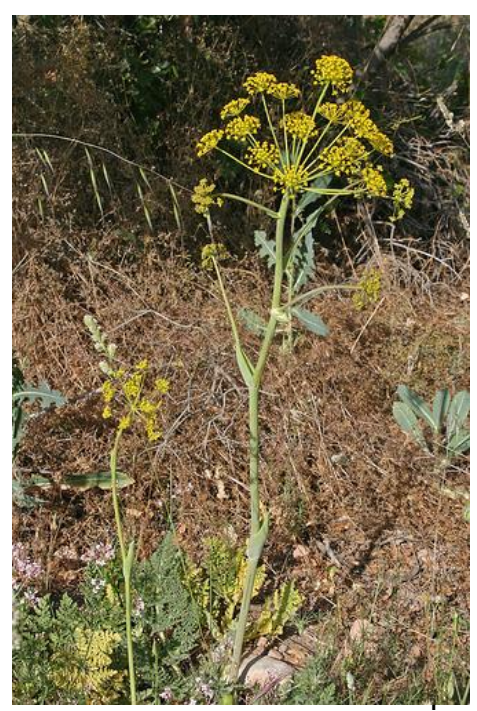

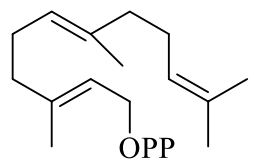

Farnesyl diphosphate

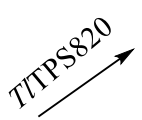

$\stackrel{T l \mathrm{TPS} 509}{\longrightarrow}$

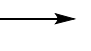

3: Guaiol
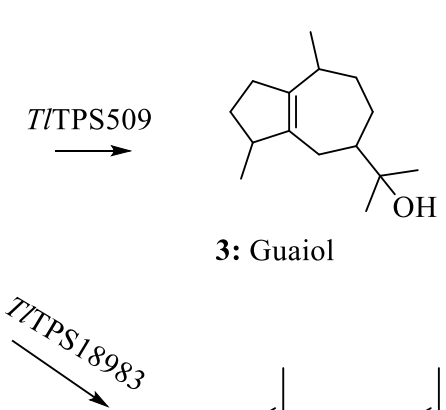

1: Epikunzeaol
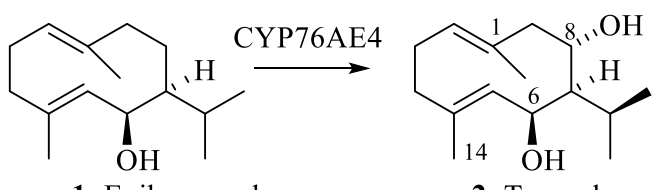

2: Tovarol

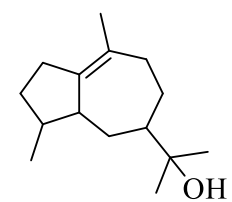

4: Bulnesol

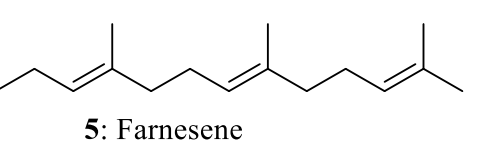




\section{Biosynthesis of tovarol and other sesquiterpenoids in Thapsia laciniata Rouy}

9

10 Trine Bundgaard Andersen ${ }^{1}$, Silas Anselm Rasmussen ${ }^{2}$, Søren Brøgger Christensen ${ }^{3}$, Henrik 11 Toft Simonsen ${ }^{2 * *}$

12 1: Department of Plant and Environmental Sciences, University of Copenhagen, Thorvaldsensvej 40, 1871

13 Frederiksberg C, Denmark

14 2: Department of Biotechnology and Biomedicine, Technical University of Denmark, Søltofts Plads 223, 2800 15 Kgs. Lyngby, Denmark

16 3: Department of Drug Design and Pharmacology, University of Copenhagen, Universitetsparken 2, 2100 17 Copenhagen, Denmark

18

$19 *$ corresponding author: hets@dtu.dk 


\section{Abstract}

22 The genus Thapsia produces a wide variety of sesquiterpenoids. The Mediterranean plant Thapsia laciniata 23 Rouy is known to have a product profile that differs from several other species in the genus. Thus, the 24 biosynthesis of sesquiterpenoids in Thapsia laciniata Rouy was investigated. Here we describe three terpene 25 synthases, $T l$ TPS820, TlTPS509 and TlTPS18983. TlTPS18983 is a multi-product enzyme with farnesene as 26 the major product, while TITPS509 produces guaiol and bulnesol along with other major and several minor 27 unknown products. TITPS820 is orthologous to TgTPS2 from Thapsia garganica L. and is an epikunzeaol 28 synthase. TgCYP76AE2 from Thapsia garganica performs a triple hydroxylation of epikunzeaol at C-13 to make dihydrocostunolide. It was therefore investigated if the cytochrome P450, TlCYP76AE4 was able to use epikunzeaol as a substrate. It was found that TICYP76AE4 hydroxylates epikunzeaol at C-8 to yield tovarol instead of dihydrocostunolide.

\section{Keywords}

33 Thapsia laciniata, Apiaceae, sesquiterpenoid, CYP76AE4, epikunzeaol, tovarol, guaiol, bulnesol 34 


\section{Introduction}

The Apiaceae family is known to include species of great nutritional and pharmacological value, such as Daucus carota L. (carrot) and Thapsia garganica L. (deadly carrot). Species from the genus Thapsia have been used in traditional medicine for millennia for a variety of ailments including rheumatism, insect bites, acne, colds and for purging (Andersen et al., 2015b). The presence of biologically active compounds, several of which are sesquiterpenoids, may justify the traditional use (Simonsen et al., 2013). While many species in Apiaceae have been studied for their chemical composition, only a few genes involved in biosynthesis of sesquiterpenoids have been characterized. The initial enzymes involved in the specialized biosynthesis of sesquiterpenoids are the sesquiterpene synthases (sqTPS). A few sqTPSs have been characterized in Apiaceae, these include a sqTPS from $D$. carota producing ( $E$ )- $\beta$-caryophyllene and $\alpha$-humulene (Yahyaa et al., 2015), a $\delta$-cadinene synthase (TgTPS1) and a epikunzeaol synthase (TgTPS2) from $T$. garganica (Pickel et al., 2012) and the germacrene D synthase from Thapsia laciniata (Andersen et al., 2015a). Of these, the epikunzeaol synthase TgTPS2 from $T$. garganica has been of particular interest. The structure of epikunzeaol makes this a likely precursor for thapsigargin. Thapsigargin is a potent inhibitor of the mammalian sarco-endoplasmic reticulum $\mathrm{Ca}^{2+}$-ATPase (Thastrup et al., 1990) with a complex structure that requires several unknown biosynthetic steps. The recently discovered cytochrome P450, TgCYP76AE2, from $T$. garganica was found to make a triple hydroxylation of epikunzeaol, thereby oxidizing C-13 to a carboxylic acid, which enabled formation of the lactone ring present in thapsigargin (Andersen et al., 2017). While several species in the Thapsia genus produce sesquiterpene lactones, these specific type of compounds are not found in T. laciniata (Drew et al., 2012).

The Thapsia genus is still in need of revision and several species are unresolved. T. laciniata Rouy has also been known as Thapsia villosa type II L. and is still listed as unresolved on http://www.theplantlist.org/. Studies of marker sequences in the Thapsia genus, however, support the assignment of T. laciniata as an independent species that is distinct from the classic T. villosa (Weitzel et al., 2014). T. laciniata is commonly found in the southern parts of France and in Catalonia, Spain. A variety of monoterpenoids and sesquiterpenoids have been reported from this species, with guaiols and germacrenols as the major sesquiterpenoids in the roots (Drew et al., 2012). In T. laciniata the only enzyme described so far is the germacrene D synthase, TlTPS7414 (Andersen et al., 2015a). Here, we present the finding of an orthologue of TgTPS2 namely $T l$ TPS 820 that also produces epikunzeaol. Two additional sqTPSs, TlTPS18983 and TlTPS509 give further insight into the diverse sesquiterpenoid profile in T. laciniata. In T. laciniata there is no equivalent sequence to $T g C Y P 76 \mathrm{AE} 2$, which explains the lack of sesquiterpene lactones. The orthologue TlCYP76AE4 instead catalyzes the hydroxylation of epikunzeaol at C-8 to yield tovarol. 


\section{Results}

\subsection{Identification of genes}

A homology-based search using BLAST was initiated to investigate if T. garganica and T. laciniata harbored orthologue genes for sesquiterpenoid biosynthesis. TgTPS1 and TgTPS2 from $T$. garganica were used as baits in the T. laciniata Illumina transcriptome dataset (Drew et al., 2013). For TgTPS2, a gene with 97.2\% nucleotide identity was discovered. The homology search for TgTPS1 did not yield any hits over 90\% in sequence identity. Three additional sqTPSs were found; the germacrene D synthase, TlTPS7414 (Andersen et al., 2015a) and the unknown TlTPS509 and TlTPS18983 (Drew et al., 2013). The data set was also mined for cytochrome P450s with high sequence similarity to $T g$ CYP76AE2, which is the gene responsible for sesquiterpene lactone formation in T. garganica. One gene was found with $92 \%$ amino acid sequence identity and 96\% nucleotide sequence identity to $T g C$ CP76AE2. The gene was named TlCYP76AE4 (Nelson, 2009). All genes were cloned into the pEAQ vector system for transformation into A. tumefaciens (Peyret and Lomonossoff, 2013). Nicotiana benthamiana was used as an expression host to examine TlTPS820, TlTPS18983, TlTPS509 and TlCYP76AE4. Samples were extracted with either hexane or by HS-SPME depending of the volatility of the enzymatic products.

\subsection{Characterization of $T$ TTPS820 expressed in $N$. benthamiana}

Leaves from $N$. benthamiana expressing TITPS820 were extracted with hexane and analyzed by GC-MS. The analysis yielded a sesquiterpenoid profile identical to $\operatorname{Tg}$ TPS2 (Figure 1). The main product of $\operatorname{Tg}$ TPS2 is epikunzeaol (1) (Figure 2), thus TlTPS820 is also an epikunzeaol synthase and an orthologue of TgTPS2. As described previously, the high injection port temperature at $250^{\circ} \mathrm{C}$ lead to cope-rearrangement of $\mathbf{1}$ (Andersen et al., 2017). Thus, 1 was partly degraded to two unknown alcohols.

\subsection{Characterization of $T I T P S 509$ expressed in $N$. benthamiana}

HS-SPME-GC-MS analysis of $N$. benthamiana leaves expressing TlTPS509 resulted in the detection of two major sesquiterpene alcohols, guaiol (3) (RI: 1585, 34\% of the area) and bulnesol (4) (RI: 1654, 20\% of the area) (Figure 3). Compound $\mathbf{3}$ and $\mathbf{4}$ exhibited similar EI MS spectrum to those of bulnesol and guaiol in NIST and Wiley GC-MS spectrum libraries. The product profile showed several additional compounds, and we could identify two minor products; guaiene (RI 1418, Rt: 8.6)) and bulnesene (RI 1487, Rt: 9.18) (Figure 3). Several peaks with a $\mathrm{m} / \mathrm{z}$ of 220 were also observed but not identified. A hexane extract of $N$. benthamiana leaves, expressing TlTPS509 was analyzed by GC-MS using a Programmed Temperature Vaporising injector (PTV) port. By using a low initial temperature of $60^{\circ} \mathrm{C}$ followed by a gradual increase to $250^{\circ} \mathrm{C}$ it was expected that thermal rearrangements were avoided. Again, $\mathbf{3}$ and $\mathbf{4}$ were detected as major compounds, but not guaiene and bulnesene, which were probably below the detection limit. Furthermore, neither of the unknown compounds with $m / z 220$ were detected in the PTV analysis. 


\subsubsection{Isolation and structure elucidation of the TITPS509 products, bulnesol and guaiol}

In order to verify the identity of $\mathbf{3}$ and $\mathbf{4}$ by NMR, the compounds were isolated in two purification steps. An initial purification of the compounds was performed on a silica column. $\mathbf{3}$ and $\mathbf{4}$ both eluted with $10 \%$ ethyl acetate in hexane and this fraction was further fractionated on a preparative GC-MS and $\mathbf{3}$ and $\mathbf{4}$ were separated and both precipitated as a colorless oil in the fraction collector. The ${ }^{1} \mathrm{H}$ and ${ }^{13} \mathrm{C}$ NMR resonance values of 3 and 4 were all in accordance with those reported by Raharivelomanana et al., (1995), which confirm the identity of $\mathbf{3}$ and $\mathbf{4}$. The spectra are provided in Supplementary data figure 12 and 13.

\subsection{Characterization of TlTPS18983 expressed in $N$. benthamiana}

Volatiles from $N$. benthamiana leaves expressing TlTPS18983 were captured by HS-SPME and analyzed by GC-MS. The analysis of these plants showed that expression of TlTPS18983 generate at least 15 products. The identification of one of the major products, farnesene (5), was based on comparison of the mass spectrum of the compound with NIST and Wiley GC-MS spectrum libraries (Figure 4, MS spectrum is provided in supplementary data Figure 3). It was not possible to identify the remaining products. Using an injection port temperature of $160{ }^{\circ} \mathrm{C}$ instead of $250{ }^{\circ} \mathrm{C}$ did not alter the composition of the chromatogram, which supports that these compounds are not a result of the GC-MS analysis method. Extraction of the products was also attempted using organic solvents such as hexane and pentane mimicking procedures that were successful for other sqTPS products. However, for TlTPS18983 we did not detect any compounds by GC-MS using organic solvent extraction.

\subsection{Characterization of $T l C Y P 76 A E 4$ using epikunzeaol from $T l T P S 820$ as substrate}

To investigate if TlCYP76AE4 would be able to use $\mathbf{1}$ as a substrate, TlTPS820 and TlCYP76AE4 were coexpressed in $N$. benthamiana. This resulted in complete conversion of $\mathbf{1}$ as seen in Figure 1. One new product was detected in the GC-MS chromatogram with an injection port temperature of $250^{\circ} \mathrm{C}$. However, the peak appeared to consist of two compounds that were co-eluting on the GC-MS. It was not possible to separate these products further. The GC-MS analysis indicated that the parent ions had a $\mathrm{m} / \mathrm{z}$ of 238 . To further support that TlTPS820 yields 1, TlCYP76AE4 was also expressed with $T g$ TPS2 and was shown to yield the same new product(s) (supplementary data Figure 1). From the chromatograms and spectrums, it was clear that $T l C Y P 76 A E 4$ yielded a new product. Co-expression of $T g$ TPS2, $T g C Y P 76 A E 2$ and TlCYP76AE4 did not result in additional new products.

\subsubsection{Isolation and structure elucidation of tovarol}

Compound 2 was isolated from the hexane extract of leaves of $N$. benthamiana expressing TlTPS820 and TlCYP76AE4. The isolation was performed by semi-preparative normal-phase HPLC and the purity of 2 was confirmed by LC-HRMS. High resolution mass spectrometry (HRMS) established the accurate mass of $\mathbf{2}$ to $m / z 261.1826\left([\mathrm{M}+\mathrm{Na}]^{+}\right)$to which the molecular formula was deduced to be $\mathrm{C}_{15} \mathrm{H}_{26} \mathrm{O}_{2}$ (calcd. for $\mathrm{C}_{15} \mathrm{H}_{26} \mathrm{O}_{2}$ 
$133[\mathrm{M}+\mathrm{Na}]^{+} \mathrm{m} / z$ 261.1825, $\left.\Delta-0.2 \mathrm{ppm}\right) .2$ was structurally elucidated by interpretation of the ${ }^{1} \mathrm{H},{ }^{13} \mathrm{C}$ and ${ }^{1} \mathrm{H}-{ }^{1} \mathrm{H}$ 134 COSY, ${ }^{1} \mathrm{H}-{ }^{13} \mathrm{C}$ ed-HSQC, ${ }^{1} \mathrm{H}^{-13} \mathrm{C}$ HMBC and ${ }^{1} \mathrm{H}-{ }^{1} \mathrm{H}$ ROESY NMR data. The data for 2 were in agreement 135 with the germacradiendiol tovarol (Figure 2), which has previously been isolated from Thapsia species (De

136 Pascual Teresa et al., 1985; Teresa et al., 1986). Due to the low quality of the published spectra and the absence 137 of ${ }^{13} \mathrm{C}$ NMR data a full structure elucidation was performed. The DEPT spectrum of the metabolite revealed 138 that 2 contained two quaternary carbon atoms, six methine carbon atoms, three methylene groups and four 139 methyl groups. Even though most of the peaks in the recorded spectrum were broad, presumably because of a 140 conformational equilibrium (Fischer et al., 1979; Triana et al., 2005), an in-depth investigation was possible 141 (Table 1). The use of 2D spectra also enabled an assignment of the signals in the ${ }^{13} \mathrm{C}$ NMR spectrum. The $E$, $E$ 142 configuration was established through a ROESY correlation between H-1 and H-5. Model building reveals 143 that only the E,E-configuration allows $\mathrm{H}-1$ and $\mathrm{H}-5$ to be near in space. ROESY correlation between $\mathrm{H}-6, \mathrm{H}-$ 14415 and H-7 revealed that H-6 and H-7 were cis-disposed. Comparison with a reference spectrum of tovarol 145 (Prof. G. Appendino personal communication) revealed that previous isolated tovarol possessed the same 146 stereochemistry at C-8 as $\mathbf{2}$, and revealed that the hydroxyl group at C-8 is $\alpha$-disposed. A ROEYS correlation 147 between $\mathrm{H}-7$ (1.05 ppm) and H-9 resonating at $1.78 \mathrm{ppm}$ revealed that this $\mathrm{H}-9$ must be $\alpha$-disposed and 148 consequently the signal at $2.49 \mathrm{ppm}$ was $\mathrm{H}-9 \beta$. The absolute configuration was assumed based on the 149 observation that sesquiterpenes from Apiaceae plants have H-7 $\alpha$-disposed (Fischer et al., 1979; Simonsen et 150 al., 2013). 2 is therefore concluded to be tovarol (De Pascual Teresa et al., 1985).

\section{Discussion}

152 A variety of sesquiterpenoids are produced in Thapsia and the sesquiterpenoid profile often differs between 153 species (Christensen et al., 1997). Previous analysis of T. garganica and T. laciniata have shown that these 154 species produce sesquiterpenoids with different backbones and side-group modifications (Drew et al., 2012).

155 T. laciniata has at least four functional sesquiterpene synthases and two of them, TlTPS18983 and TlTPS509, 156 are multi-product synthases. Compared to the two sqTPS found in T. garganica this correlates well with the 157 higher number of metabolites described in T. laciniata (Christensen et al., 1997; Drew et al., 2012, 2013). In 158 Daucus carota 20 sqTPS (TPS-a family) are predicted, which show that even between closely related species 159 the number of sqTPS's can vary tremendously (Banasiak et al., 2016; Keilwagen et al., 2017).

160 Several farnesene synthases have been described in literature. $T l 18983$ is however the first farnesene synthase 161 from Apiaceae. The finding of a farnesene synthase is supported by the previous detection of $\beta$-farnesene in 162 T. laciniata (Drew et al., 2012). It is interesting that $T l 18983$ is able to produce a large number of products, 163 which cannot merely be characterized as minor side products, at least judging by expression in N. benthamiana. 164 Analyzing a multiproduct sqTPS is complex due to the difficulty of separating the products by GC-MS, and 165 due to very volatile nature of these compounds. Sesquiterpenoids in many species are a part of the volatile 166 mixture of compounds that is emitted by the plants. It can be hypothesized that the products produced by 
TlTPS18983 are a part of the volatile signaling compounds of T. laciniata, as also seen in other plants (Flamini et al., 2003; Máday et al., 1999; Wang et al., 2015).

Two major products of TlTPS509 were identified as guaiol (3) and bulnesol (4). Previous investigation of $T$. laciniata sesquiterpenoids (Drew et al., 2012; Lemmich et al., 1984) also showed that 3 is produced by this species. Three guaiane esters, $(4 S, 5 S, 7 S, 8 S)$-8-p-Coumaroyloxy-1(10)-guaiene-11-ol, $(4 S, 5 S, 7 S, 8 S)$-8Feruloyloxy-1(10)-guaiene-11-ol, and (4S, 5S, 7S, 8S)-8-Senecioyloxy-1(10)-guaien-11-ol have also been found in T. laciniata (Lemmich et al., 1991). All of these are derived from the structure of $\mathbf{4}$ and have different side chain modifications at C-8. This indicates that bulnesol is a possible precursor of these three structures. The described modifications at C-8 are expected to involve a hydroxylation at C-8 followed by the addition of side chains by acyl transferases. Currently, T. laciniata is the only species in the genus Thapsia that has been found to produce guaiols (Christensen et al., 1997). Furthermore, $\alpha$-guaiene was previously found in $T$. laciniata (Drew et al., 2012). This is a minor product of TITPS509 and is the non-hydroxylated compound with the same type of 5- and 7-ring structure as guaiols. The same is also observed for bulnesene and bulnesol. Co-expression of CYP76AE4 and TlTPS509 did not provide any new products. Thus, an orthologue of CYP76AE4 might be responsible for the hydroxylation at C-8, though this remains to be examined.

TlTPS820 produces epikunzeaol (1) as a main product. The discovery of the two orthologues sqTPS genes (TgTPS2 and TITPS820) in T. laciniata and T. garganica shows that the epikunzeaol synthase can serve as a marker for the Thapsia genus. It also shows that the species within Thapsia have evolved different downstream products by using 1 as a substrate. While TgCYP76AE2 and TlCYP76AE4 are both able to use 1 as a substrate, they perform hydroxylation on different carbon positions. This difference is also reflected in the metabolite profiles of the two species. In contrast to most Thapsia species, T. laciniata does not contain guaianolides, for which epidihydrocostunolide (the product of TgCYP76AE2 in T. garganica) appears to be a precursor (Andersen et al., 2017). Tovarol has currently not been detected from T. laciniata and could be involved in the biosynthesis of a downstream product yet to be discovered. However, tovarol and several derivatives was isolated from the closely related Thapsia minor Hoffmanns. \& Link (De Pascual Teresa et al., 1985). It is likely that similar biosynthetic routes are present in $T$. laciniata and $T$. minor since these two species are very closely related (Banasiak et al., 2016; Weitzel et al., 2014). It remains to be investigated if T. minor harbors orthologue genes to those described here and in T. garganica.

The products detected by GC-MS from TgCYP76AE2 were shown to be Cope re-arrangements of dihydrocostunolide (Andersen et al., 2017). It is highly probable that $\mathbf{2}$ from TlCYP76AE4 has also undergone Cope re-arrangement during exposure to the high injection port temperature in the GC-MS (Figure 1). Through the isolation of $\mathbf{2}$ it was clear from our LC data that TlCYP76AE4 only produces 2 as the product. 
The expression of genes, involved in sesquiterpene biosynthesis in $N$. benthamiana has previously been reported to result in the conjugation of produced sesquiterpenoids. This was shown for artemisinic acid in the artemisinin pathway, which was conjugated to a diglucoside (van Herpen et al., 2010). In costunolide biosynthesis, the analysis was affected by the conjugation of costunolide to glutathione or cysteine (Liu et al., 2011). LC-MS analysis of 2 from TlCYP76AE4 did not reveal any conjugation to the two alcohol groups, which therefore allowed for detection by GC-MS as well. 1 on the other hand from TlTPS820 and $\operatorname{Tg}$ TPS2 was found both in its free form in GC-MS analysis and as a disaccharide in LC-MS analysis.

\section{Conclusion}

We have described the activity of three sesquiterpene synthases from Thapsia laciniata. TlTPS820 was found to be an epikunzeaol synthase whereas TlTPS18983 and TlTPS509 are multi-product sesquiterpene synthases, with farnesene as the major product of TlTPS18983 and guaiol and bulnesol as the major products of TlTPS509. TlTPS820 is an orthologue to TgTPS2 from Thapsia garganica and co-expression with the cytochrome P450 TlCYP76AE4 led to the biosynthesis of tovarol. Thus, we have shown that TlCYP76AE4 hydroxylate epikunzeaol at C-8 to yield tovarol.

\section{Experimental section}

\subsection{Plant material}

Thapsia laciniata Rouy, Apiaceae (Banasiak et al., 2016; Weitzel et al., 2014) roots were collected in early June 2008, just west of Cannes, France (GPS 43.540958, 6.816158). Voucher specimens of T. laciniata (HTS 2008-01) are stored at herbarium C (Natural History Museum of Denmark, Copenhagen).

\subsection{Identification and cloning of genes}

The sesquiterpene synthases TlTPS18983 (MG680745), TlTPS509 (MG680746) and TlTPS820 (MG680744) were found in a transcriptome dataset from T. laciniata root (SRX252523) (Drew et al., 2013). The full-length sequences were obtained from a cDNA library from $T$. laciniata root material. The discovery of $T g$ TPS2 (JQ290345) and TgCYP76AE2 (KX826943) has been presented previously (Andersen et al., 2017; Pickel et al., 2012). $T g C Y P 76 A E 2$ was blasted into the transcriptome dataset to search for a homologues sequence. The resulting P450 was named, TlCYP76AE4 (MG680747) (Nelson, 2009).

Forward and reverse primers for all genes were designed with USER-overhangs, to enable cloning into a pEAQ-USER compatible version of the pEAQ-HT vector (Supplementary data, table 1) (Luo et al., 2016). 
pEAQ-HT harbors the viral suppressor p19 and was kindly provided by George Lomonosonoff (John Innes 230 Research Centre, Norwich, UK) (Peyret and Lomonossoff, 2013). USER cloning was performed as previously 231 depicted (Nour-Eldin et al., 2006).

\subsection{Expression of $T l T P S 18983, T l T P S 509 T g$ TPS2, TlTPS802, TgCYP76AE2 and TlCYP76AE4 in Nicotiana benthamiana}

Nicotiana benthamiana plants were grown from seeds at $24{ }^{\circ} \mathrm{C} / 19{ }^{\circ} \mathrm{C}$ (day/night) for 5 weeks before transformation. The transformation of Agrobacterium tumefaciens and infiltration of $N$. benthamiana with A. tumefaciens was achieved as previously described (Bach et al., 2014). Plants were harvested 5 days after infiltration. In short, $10 \mathrm{ml}$ LB containing kanamycin, rifampicin and carbenicillin was inoculated with several agrobacterium colonies containing the plasmid of interest. Cultures were grown overnight at $28{ }^{\circ} \mathrm{C}$ and 200 rpm. Cell pellets were washed twice with water before a final resuspension in water followed by a dilution to $\mathrm{OD}_{600}$ 0.5. Resuspended A. tumefaciens carrying plasmids containing AttHMGR, TlTPS or TlCYP76AE4 were mixed 1:1:1 and infiltrated into leaves of at least three $N$. benthamiana plants by use of a syringe. Plants were placed at $24^{\circ} \mathrm{C} / 19^{\circ} \mathrm{C}$ (day/night) and harvested five days after infiltration. As controls, plants were infiltrated with A. tumefaciens carrying plasmids with AttHMG, or AttHMGR plus TlTPSs.

The $\sim 100$ plants needed for purification of tovarol were infiltrated by use of vacuum. Three A. tumefaciens cultures containing AttHMGR, TlTPS820 or TlCYP76AE4 were grown overnight at $28^{\circ} \mathrm{C}$ and $200 \mathrm{rpm}$ in 500 $\mathrm{ml} \mathrm{LB}$ (containing kanamycin, rifampicin and carbenicillin) from $20 \mathrm{ml}$ starter cultures. Cell pellets were washed twice with water before final resuspension in water followed by a dilution to $\mathrm{OD}_{600} 0.5$. Resuspended A. tumefaciens carrying plasmids containing AttHMGR, TlTPS820 or TlCYP76AE4 were mixed 1:1:1. Plants were submerged in a $1 \mathrm{~L}$ suspension of $A$. tumefaciens and infiltrated by use of vacuum at 50-100 mbar for 1 min (Andersen-Ranberg et al., 2016).

\subsection{GC-MS detection of sesquiterpenoids from liquid extractions}

Two leaf discs (diameter $3 \mathrm{~cm}$ ) from $N$. benthamiana, expressing genes from $T$. laciniata were extracted with $1.2 \mathrm{ml}$ hexane for GC-MS analysis to provide one sample, a minimum of three biological replicates were examined. Samples were analyzed on a Shimadzu GCMS-QP2010. The column used was an Agilent HP-5MS UI, $20 \mathrm{~m}, 0.18 \mathrm{~mm}$ diameter $\times 0.18 \mu \mathrm{m}$ film thickness. The pressure was kept at $66.7 \mathrm{kPa}$ giving a column flow of $1 \mathrm{~mL} / \mathrm{min}$. The injection port temperature was set to $250{ }^{\circ} \mathrm{C}$. The oven temperature was set to $60{ }^{\circ} \mathrm{C}$ for $3 \mathrm{~min}$, and then increased to $160{ }^{\circ} \mathrm{C}$ with a rate of $7{ }^{\circ} \mathrm{C} / \mathrm{min}$. The temperature was further increased to 300 ${ }^{\circ} \mathrm{C}$ at a rate of $50{ }^{\circ} \mathrm{C} / \mathrm{min}$, held for $5 \mathrm{~min}$, finally increased to $320^{\circ} \mathrm{C}$ at $50{ }^{\circ} \mathrm{C} / \mathrm{min}$ and maintained for $3 \mathrm{~min}$. 
261 Shimadzu software Lab Solutions, GCMS Solutions version 2.50 SU3, with the 2008 libraries provided by 262 NIST and Wiley.

\subsection{HS-SPME-GC-MS analysis of sesquiterpenoids}

$T l$ TPS18983 and TlTPS509 were also analyzed on GC-MS by use of HS-SPME fibers. HS-SPME fibers were purchased from Supelco Sigma-Aldrich, Denmark. A whole leaf was placed in a $20 \mathrm{ml}$ glass vial and extracted at $60{ }^{\circ} \mathrm{C}$ for $20 \mathrm{~min}$ with a SPME fiber exposed to the air above the leaf (Andersen et al., 2015a). Immediately hereafter, the SPME fiber was run on the GC-MS. Samples were analyzed on a Shimadzu GCMS-QP2010. The column used was an Agilent HP-5MS UI, $20 \mathrm{~m}, 0.18 \mathrm{~mm}$ diameter $\times 0.18 \mu \mathrm{m}$ film thickness. The pressure was kept at $66.7 \mathrm{kPa}$ giving a column flow of $1 \mathrm{~mL} / \mathrm{min}$. The injection port temperature was set to $160{ }^{\circ} \mathrm{C}$ or $250{ }^{\circ} \mathrm{C}$. The oven temperature was set to $60{ }^{\circ} \mathrm{C}$ for $3 \mathrm{~min}$, and then increased to $160^{\circ} \mathrm{C}$ with a rate of $7{ }^{\circ} \mathrm{C} / \mathrm{min}$. The temperature was further increased to $300{ }^{\circ} \mathrm{C}$ at a rate of $50{ }^{\circ} \mathrm{C} / \mathrm{min}$, held for $5 \mathrm{~min}$, finally increased to $320{ }^{\circ} \mathrm{C}$ at $50{ }^{\circ} \mathrm{C} / \mathrm{min}$ and maintained for $3 \mathrm{~min}$. The carrier gas was $\mathrm{H}_{2}$ and the ionization electron energy was $70 \mathrm{eV}$. The ion source temperature was $230{ }^{\circ} \mathrm{C}$ with an interface temperature $280{ }^{\circ} \mathrm{C}$. The total run time was $28.49 \mathrm{~min}$. All data were analyzed using the Shimadzu software Lab Solutions, GCMS Solutions version 2.50 SU3, with the 2008 libraries provided by NIST and Wiley.

\subsection{Analytical LC-MS detection of sesquiterpenoids}

Two leaf discs (diameter $3 \mathrm{~cm}$ ) were ground in liquid nitrogen. Samples were extracted with $1200 \mu 180 \%$ methanol and sonicated for $30 \mathrm{~min}$. Before LC-MS analysis samples were filtered through a $0.45 \mu \mathrm{m}$ filter. Analytical LC-MS was carried out using an Agilent 1100 Series LC (Agilent Technologies, Santa Clara, CA, USA) coupled to a Bruker HCT-Ultra ion trap mass spectrometer (Bruker Daltonics, Bremen, Germany). Separation was achieved on a Gemini-NX $\mathrm{C}_{18}$ column (Phenomenex; $2 \times 150 \mathrm{~mm}, 3 \mu \mathrm{m}$ ) maintained at $35^{\circ} \mathrm{C}$. 282 The mobile phases were: A, water with $0.1 \%$ (v/v) $\mathrm{HCOOH}$; B, acetonitrile with $0.1 \%(\mathrm{v} / \mathrm{v}) \mathrm{HCOOH}$. The 283 gradient program was: 0 to $1 \mathrm{~min}$, isocratic $12 \% \mathrm{~B} ; 1$ to $33 \mathrm{~min}$, linear gradient 12 to $80 \% \mathrm{~B} ; 33$ to $35 \mathrm{~min}$, 284 linear gradient 80 to $99 \% \mathrm{~B} ; 35$ to $38 \mathrm{~min}$, isocratic $99 \% \mathrm{~B} ; 38-47 \mathrm{~min}$, isocratic $12 \% \mathrm{~B}$. The flow rate was $0.2 \mathrm{~mL} / \mathrm{min}$. The mass spectrometer was operated in positive mode and the range $\mathrm{m} / \mathrm{z} 100-1000$ was acquired.

High-resolution mass spectrometry of $\mathbf{2}$ was acquired on a maXis HD mass spectrometer (Bruker Daltronics, 287 Bremen, Germany) coupled to an Ultima 3000 series HPLC-DAD (Thermo, Waltham, Massachusetts, USA). 288 Separation was achieved on a Kinetex $\mathrm{C}_{18}$ column (Phenomenex, Torrance, USA, $150 \times 2 \mathrm{~mm}, 2.6 \mu \mathrm{m}$ ) using 289 a linear gradient consisting of $\mathrm{A}, \mathrm{H}_{2} \mathrm{O}$ with $20 \mathrm{mM} \mathrm{HCOOH}$ and $\mathrm{B}$, HPLC-MS grade MeCN with $20 \mathrm{mM}$ $290 \mathrm{HCOOH}$, from $10-100 \% \mathrm{~B}$ in $10 \mathrm{~min}$ with a flow rate of $0.4 \mathrm{ml} / \mathrm{min}$, the column was maintained at $40{ }^{\circ} \mathrm{C}$. 291 The mass spectrometer was operated in positive ESI acquiring the $m / z$ range of $75-1250$. 
293 For isolation of guaiol and bulnesol, a large-scale hexane extraction was made from $N$. benthamiana leaves 294 expressing truncated AtHMGR and TlTPS509. The leaves from approximately 100 five-week-old plants were 295 extracted with hexane. The crude hexane extract was subjected to an initial preparative separation on a silica 296 column and eluted stepwise in hexane-ethyl acetate, from hexane to hexane:ethyl acetate (80:20) with $2 \%$ 297 increments. The final purification of products from TlTPS509 were performed on a preparative GC-MS using 298 an Agilent 7890B GC installed with an Agilent 5977A inert MSD, GERSTEL Preparative Fraction Collector 299 (PFC) AT 6890/7890 and a GERSTEL CIS 4C Bundle injection port. For separation by GC, a RESTEK Rtx3005 column $(30 \mathrm{~m} \times 0.53 \mathrm{~mm}$ ID $\times 1 \mu \mathrm{m} \mathrm{df})$ with $\mathrm{H}_{2}$ as the carrier gas was used. A split piece with a split of 1:100 301 (MS:PFC) was placed at the end of this column. Sufficient amounts of sesquiterpene product for NMR analysis $302(0.5-1 \mathrm{mg})$ were obtained with $5 \mu \mathrm{L}$ injections of extract repeated 100 times. The injection port was put in 303 solvent vent mode with a carrier gas flow of $100 \mathrm{~mL} / \mathrm{min}$ until minute 0.17 , combined with an injection speed 304 of $1.5 \mathrm{~mL} / \mathrm{min}$. The purge flow was set to $3 \mathrm{~mL} / \mathrm{min}$ from minute 0.17 to 2.17 . The injection temperature was 305 held at $40{ }^{\circ} \mathrm{C}$ for $0.1 \mathrm{~min}$ followed by ramping at $12{ }^{\circ} \mathrm{C} / \mathrm{sec}$ until $320^{\circ} \mathrm{C}$, which was then held for $2 \mathrm{~min}$. The 306 column flow was set to $7.5 \mathrm{~mL}$, which was held constant throughout the GC program. The GC program was 307 set to hold at $60{ }^{\circ} \mathrm{C}$ for $1 \mathrm{~min}$, ramp $20{ }^{\circ} \mathrm{C} / \mathrm{min}$ to $320^{\circ} \mathrm{C}$ and $320{ }^{\circ} \mathrm{C}$ held for $3 \mathrm{~min}$. The temperature of the 308 transfer line from GC to PFC and the PFC itself was set to $250{ }^{\circ} \mathrm{C}$. The PFC was set to collect the selected 309 peaks based on the MS spectrum. The MS was set in scan mode from $\mathrm{m} / \mathrm{z} 35$ to $\mathrm{m} / \mathrm{z} 500$, with a threshold of 310 150. Solvent cut-off was set to $4 \mathrm{~min}$, and the temperature of the MS source and the MS quadrupole was set to $311300{ }^{\circ} \mathrm{C}$ and $150{ }^{\circ} \mathrm{C}$, respectively. Traps were kept at $-20^{\circ} \mathrm{C}$, in case products were prone to rearrangement, and 312 rinsed with chloroform-d (Euriso-top, 99.8 atom \% D).

\subsection{Isolation of tovarol from TlCYP76AE4 by HPLC}

314 For isolation of tovarol, a large-scale hexane extraction was made from $N$. benthamiana leaves expressing 315 truncated AtHMGR, TlTPS820 and TlCYP76AE4. The leaves from approximately 100 five-week-old plants 316 were extracted with hexane. The crude hexane extract was subjected to an initial preparative separation on a 317 Isolera autoflasher using a $10 \mathrm{~g}$ diol functionalized silica column (Biotage, Uppsala, Sweden) eluted stepwise 318 in hexane-ethyl acetate, from hexane to hexane:ethyl acetate (80:20) with $2 \%$ increments. Final isolation of 319 tovarol was achieved by semi-preparative HPLC utilizing a $250 \times 10 \mathrm{~mm}, 7 \mu \mathrm{m}$ Nucleosil PEI column 320 (Macherey-Nagel) eluted in a linear hexane-isopropanol gradient from 0-5\% isopropanol in $20 \mathrm{~min}$, on a 321 Waters 600 HPLC equipped with a Waters 996 PDA detector. The purity was confirmed by LC-MS as described in 5.6. 
NMR-spectra were acquired using a $600 \mathrm{MHz}$ Bruker Avance III HD NMR spectrometer $\left({ }^{1} \mathrm{H}\right.$ operating frequency $600.13 \mathrm{MHz}$ ) equipped with a Bruker SampleJet sample changer and a cryogenically cooled gradient inverse triple-resonance 1.7-mm TCI probe-head (Bruker Biospin, Rheinstetten, Germany) optimized for ${ }^{13} \mathrm{C}$ and ${ }^{1} \mathrm{H}$. Samples were analyzed at $300 \mathrm{~K}$. Proton spectra, at $600.03 \mathrm{MHz}$, were obtained using $30^{\circ}$-pulses, a spectral width of $12 \mathrm{kHz}$, collecting 16 scans with a length of 65536 data points with a relaxation delay of 1.0 sec. Carbon spectra were acquired at $150.88 \mathrm{MHz}$ with $30^{\circ}$-pulses, a spectral width of $36 \mathrm{kHz}$, collecting 256 scans with a length of 65536 data points and with a relaxation delay of $2.0 \mathrm{sec}$. The ${ }^{13} \mathrm{C}$ nuclei were ${ }^{1} \mathrm{H}-$ 331 decoupled using the Waltz-16 composite pulse-decoupling scheme. FID's were exponentially multiplied with 332 a line-broadening factor of $1.0 \mathrm{~Hz}$ before Fourier transformation. The 2D experiments were recorded using 333 Bruker standard parameter settings. The isolated tovarol was dissolved in $\mathrm{MeCN}^{-\mathrm{d}_{3}}(99.8$ atom \% D) prior to 334 NMR analysis.

\section{Acknowledgements}

Trine Bundgaard Andersen, Henrik Toft Simonsen and Søren Brøgger Christensen were supported by SPOTLight, a grant from the Danish Council for Strategic Research. George Lomonosonoff (John Innes Research Centre, Norwich, UK) provided the pEAQ-HT plasmid. NMR equipment used in this work was purchased via grant \#10-085264 from The Danish Research Council for Independent Research | Nature and Universe. We thank Professori Ordinari Giovanni Battista Appendino, Università degli Studi del Piemonte 
346 Figure 1: GC-MS chromatogram of hexane extracts from $N$. benthamiana expressing AttHMGR+TlTPS820, 347 AttHMGR+TgTPS2, and AtHMGR+TlTPS820 + TlCYP76AE4. The chromatograms show that $T g$ TPS2 and $348 T l$ TPS820 both produce epikunzeaol (1) as previously published for TgTPS2 (Andersen et al., 2017), and with 349 co-expression of TlCYP76AE4 this product disappear and a cope rearranged product of tovarol (2) is found. 350 This confirms the functionality of both enzymes.

351 Figure 2: The biosynthetic routes catalyzed by the four described Thapsia laciniata enzymes TlTPS820, $352 T l$ TPS509, $T l$ TPS18983, and TlCYP76AE4 leading to tovarol (2).

353 Figure 3: HS-SPME GC-MS analysis of TlTPS509 expressed together AtHMGR, the control shows the 354 expression of $A t \mathrm{HMGR}$ alone. Guaiol (3) is seen as the major product (Rt $9.89 \mathrm{~min}$ ), with bulnesol (4), Rt: 355 10.2, a-guaiene (Rt: 8.6), and a-bulnesene (Rt: 9.18) as minor products. * show unidentified sesquiterpene like 356 products, which is either ketone formed during the biosynthesis or breakdown products of the alcohols formed 357 during the GC-MS analysis. Based on this TlTPS509 is a guaiol synthase. MS spectra are presented in 358 supplementary data

359 Figure 4: a) HS-SPME GC-MS analysis of TlTPS18983 expressed together AtHMGR, the control sample 360 show the expression of AtHMGR alone. farnesene (5) is seen as the major product (Rt $8.75 \mathrm{~min}$ ), * show 361 unidentified sesquiterpene like products. Based on this TlTPS18983 is a multiproduct terpene synthase with 362 farnesene as the major product. MS spectra are presented in supplementary data. 
365 Table 1: NMR data for tovarol (2). The spectrum was recorded in $\mathrm{CD}_{3} \mathrm{CN}$ at $600 \mathrm{MHz}$. Most of the signals are 366 broad, which is probably because of a conformational equilibrium. Numbering is shown in Figure 2.

\begin{tabular}{|c|c|c|}
\hline Position & $\delta_{\mathrm{H}}$ mult $(\mathrm{J} \mathrm{Hz})$ & $\delta_{\mathrm{C}}$ type \\
\hline 1 & 4.96, br.qd $(J 12.0,5.1)$ & $131.3 \mathrm{CH}$ \\
\hline $2 \beta$ & $2.39, \mathrm{dd},(J 12.0,5.1)$ & $25.6 \mathrm{CH}_{2}$ \\
\hline $2 \alpha$ & $2.06, \mathrm{ddt},(J 12.0,5.1,2.0)$ & \\
\hline \multirow[t]{2}{*}{3} & 2.14, br.dd, $(J 12.0,5.1)$ & $39.7 \mathrm{CH} 2$ \\
\hline & 2.06, br.ddt, $(J 12.0,5.1,2.0)$ & \\
\hline 4 & & $132.3 \mathrm{C}$ \\
\hline 5 & 5.13, br.d (J 6.6) & $133.6 \mathrm{CH}$ \\
\hline 6 & 4.57, br.d (J 6.6) & $69.2 \mathrm{CH}$ \\
\hline 7 & 1.05 , overlaid & $54.7 \mathrm{CH}$ \\
\hline 8 & 4.16 , br.ddd, $(J 11.7,5.9,0.7)$ & $73.2 \mathrm{CH}$ \\
\hline $9 \beta$ & $2.49, \mathrm{dd}(J 11.9,6.0)$ & $45.6 \mathrm{CH}_{2}$ \\
\hline $9 \alpha$ & $1.78, \mathrm{t}(J 11.9)$ & \\
\hline 10 & & $135.5 \mathrm{C}$ \\
\hline 11 & $2.00 \mathrm{dq},(J 10.3,6.6)$ & $26.4 \mathrm{CH}$ \\
\hline 12 & $1.02, \mathrm{~d},(\mathrm{~J} 6.6)$ & $21.9 \mathrm{CH}_{3}$ \\
\hline 13 & $1.05, \mathrm{~d},(J 6.2)$ overlaid & $21.4 \quad \mathrm{CH}_{3}$ \\
\hline 14 & $1.64, \mathrm{~s}$ & $24.2 \mathrm{CH}_{3}$ \\
\hline 15 & $1.42, \mathrm{~s}$ & $16.8 \mathrm{CH}_{3}$ \\
\hline $\mathrm{OH}$ & 2.14 , br.s overlaid & \\
\hline
\end{tabular}




\section{References}

Andersen-Ranberg, J., Kongstad, K.T., Nielsen, M.T., Jensen, N.B., Pateraki, I., Bach, S.S., Hamberger, B., Zerbe, P., Staerk, D., Bohlmann, J., Møller, B.L., Hamberger, B., 2016. Expanding the landscape of diterpene structural diversity through stereochemically controlled combinatorial biosynthesis. Angew. Chemie - Int. Ed. 55, 2142-2146.

Andersen, T.B., Cozzi, F., Simonsen, H.T., 2015a. Optimization of biochemical screening methods for volatile and unstable sesquiterpenoids using HS-SPME-GC-MS. Chromatography 2, 277-292.

Andersen, T.B., Lopez, C.Q., Manczak, T., Martinez, K.A., Simonsen, H.T., 2015b. Thapsigargin - from Thapsia L. to Mipsagargin. Molecules 20, 6113-6127.

Andersen, T.B., Martinez, K.A., Rasmussen, S.A., Boughton, B.A., Jørgensen, K., Andersen-Ranberg, J., Nyberg, N., Christensen, S.B., Simonsen, H.T., 2017. Localization and in-vivo characterization of Thapsia garganica CYP76AE2 indicates a role in thapsigargin biosynthesis. Plant Physiol. 174, 56-72.

Bach, S.S., Bassard, J.-É., Andersen-Ranberg, J., Møldrup, M.E., Simonsen, H.T., Hamberger, B., 2014. Highthroughput testing of terpenoid biosynthesis candidate genes using transient expression in Nicotiana benthamiana, in: Rodríguez-Concepción, M. (Ed.), Plant Isoprenoids, Methods in Molecular Biology. Springer New York, New York, USA, pp. 245-255.

Banasiak, Ł., Wojewódzka, A., Baczyński, J., Reduron, J.-P., Piwczyński, M., Kurzyna-Młynik, R., Gutaker, R., Czarnocka-Cieciura, A., Kosmala-Grzechnik, S., Spalik, K., 2016. Phylogeny of Apiaceae subtribe Daucinae and the taxonomic delineation of its genera. Taxon $65,563-585$.

Christensen, S.B., Andersen, A., Smitt, U.W., 1997. Sesquiterpenoids from Thapsia species and medicinal chemistry of the thapsigargins. Fortschritte der Chemie Org. Naturstoffe. Prog. Chem. Org. Nat. Prod. $71,129-167$.

De Pascual Teresa, J., Morán, J.R., Hernández, J.M., Grande, M., 1985. Tovarol and other germacrane derivatives from Thapsia villosa. Phytochemistry 24, 1779-1783.

Drew, D.P., Dueholm, B., Weitzel, C., Zhang, Y., Sensen, C.W., Simonsen, H.T., 2013. Transcriptome analysis of Thapsia laciniata Rouy provides insights into terpenoid biosynthesis and diversity in Apiaceae. Int. J. Mol. Sci. 14, 9080-9098.

Drew, D.P., Rasmussen, S.K., Avato, P., Simonsen, H.T., 2012. A comparison of headspace solid-phase microextraction and classic hydrodistillation for the identification of volatile constituents from Thapsia spp. provides insights into guaianolide biosynthesis in Apiaceae. Phytochem. Anal. 23, 44-51. 
Fischer, N.H., Olivier, E.J., Fischer, H.D., 1979. The biogenesis and chemsitry of sesquiterpene lactones. Fortschritte der Chemie Org. Naturstoffe. Prog. Chem. Org. Nat. Prod. 38, 47-390.

Flamini, G., Cioni, P.L., Morelli, I., 2003. Differences in the fragrances of pollen, leaves, and floral parts of garland (Chrysanthemum coronarium) and composition of the essential oils from flowerheads and leaves. J. Agric. Food Chem. 51, 2267-2271.

Keilwagen, J., Lehnert, H., Berner, T., Budahn, H., Nothnagel, T., Ulrich, D., Dunemann, F., 2017. The terpene synthase gene family of carrot (Daucus carota L.): Identification of QTLs and candidate genes associated with terpenoid volatile compounds. Front. Plant Sci. 8, 1930.

Lemmich, E., Jensen, B., Rasmussen, U., 1984. (8R,14S)-8-angeloyloxythapsan-14-ol, a sesquiterpene with a novel carbon skeleton, from Thapsia villosa. Phytochemistry 23, 809-811.

Lemmich, E., Wagner Smitt, U., Sandholm Jensen, J., Brøgger Christensen, S., 1991. Guaiane esters from Thapsia villosa. Phytochemistry 30, 2987-2990.

Liu, Q., Majdi, M., Cankar, K., Goedbloed, M., Charnikhova, T., Verstappen, F.W.A., de Vos, R.C.H., Beekwilder, J., van der Krol, S., Bouwmeester, H.J., 2011. Reconstitution of the costunolide biosynthetic pathway in yeast and Nicotiana benthamiana. PLoS One 6, e23255.

Luo, D., Callari, R., Hamberger, B., Wubshet, S.G., Nielsen, M.T., Andersen-Ranberg, J., Hallstrom, B.M., Cozzi, F., Heider, H., Lindberg Moller, B., Staerk, D., Hamberger, B., 2016. Oxidation and cyclization of casbene in the biosynthesis of Euphorbia factors from mature seeds of Euphorbia lathyris L. Proc. Natl. Acad. Sci. U. S. A. 113, E5082-9.

Máday, E., Szöke, É., Muskáth, Z., Lemberkovics, É., 1999. A study of the production of essential oils in chamomile hairy root cultures. Eur. J. Drug Metab. Pharmacokinet. 24, 303-308.

Nelson, D., 2009. The Cytochrome P450 Homepage. Hum. Genomics 4, 59-65.

Nour-Eldin, H.H., Hansen, B.G., Nørholm, M.H.H., Jensen, J.K., Halkier, B.A., 2006. Advancing uracilexcision based cloning towards an ideal technique for cloning PCR fragments. Nucleic Acids Res. 34, e122.

Peyret, H., Lomonossoff, G.P., 2013. The pEAQ vector series: the easy and quick way to produce recombinant proteins in plants. Plant Mol. Biol. 83, 51-58.

Pickel, B., Drew, D.P., Manczak, T., Weitzel, C., Simonsen, H.T., Ro, D., 2012. Identification and characterization of a kunzeaol synthase from Thapsia garganica: Implications for the biosynthesis of the 
pharmaceutical thapsigargin. Biochem. J. 448, 261-271.

Raharivelomanana, P., Bianchini, J.-P., Cambon, A., Azzaro, M., Faure, R., 1995. Two-dimensional NMR of sesquiterpenes. 8 - complete assignment of $1 \mathrm{H}$ and 13C NMR spectra of seven sequiterpene alcohols from Neocallitropsis pancheri. Magn. Reson. Chem. 33, 233-235.

Simonsen, H.T., Weitzel, C., Christensen, S.B., 2013. Guaianolide sesquiterpenoids - Their pharmacology and biosynthesis, in: Ramawat, K.G., Merillon, J.M. (Eds.), Handbook of Natural Products - Phytochemistry, Botany and Metabolism of Alkaloids, Phenolics and Terpenes, Handbook of Natural Products. SpringerVerlag, Berlin, Germany, pp. 3069-3098.

Thastrup, O., Cullen, P.J., Drobak, B.K., Hanley, M.R., Dawson, A.P., 1990. Thapsigargin, a tumor promoter, discharges intracellular $\mathrm{Ca} 2+$ stores by specific inhibition of the endoplasmic reticulum $\mathrm{Ca} 2(+)$-ATPase. Proc. Natl. Acad. Sci. U. S. A. 87, 2466-2470.

Triana, J., López, M., Pérez, F.J., González-Platas, J., Quintana, J., Estévez, F., León, F., Bermejo, J., 2005. Sesquiterpenoids from Pulicaria canariensis and their cytotoxic activities. J. Nat. Prod. 68, 523-531.

van Herpen, T.W., Cankar, K., Nogueira, M., Bosch, D., Bouwmeester, H.J., Beekwilder, J., 2010. Nicotiana benthamiana as a production platform for artemisinin precursors. PLoS One 5, e14222.

Wang, G.P., Yu, X.D., Fan, J., Wang, C.S., Xia, L.Q., 2015. Expressing an (E)-beta-farnesene synthase in the chloroplast of tobacco affects the preference of green peach aphid and its parasitoid. J. Integr. Plant Biol.

Weitzel, C., Rønsted, N., Spalik, K., Simonsen, H.T., 2014. Resurrecting deadly carrots: Towards a revision of Thapsia (Apiaceae) based on phylogenetic analysis of nrITS sequences and chemical profiles. Bot. J. Linn. Soc. 174.

Yahyaa, M., Tholl, D., Cormier, G., Jensen, R., Simon, P.W., Ibdah, M., 2015. Identification and characterization of terpene synthases potentially involved in the formation of volatile terpenes in carrot (Daucus carota L.) roots. J. Agric. Food Chem. 63, 4870-4878. 